\title{
CRITICAL CHROMATIC NUMBER AND THE COMPLEXITY OF PERFECT PACKINGS IN GRAPHS
}

\author{
DANIELA KÜHN AND DERYK OSTHUS
}

\begin{abstract}
Let $H$ be any non-bipartite graph. We determine asymptotically the minimum degree of a graph $G$ which ensures that $G$ has a perfect $H$-packing. More precisely, we determine the smallest number $\tau$ having the following property: For every positive constant $\gamma$ there exists an integer $n_{0}=n_{0}(\gamma, H)$ such that every graph $G$ whose order $n \geq n_{0}$ is divisible by $|H|$ and whose minimum degree is at least $(\tau+\gamma) n$ contains a perfect $H$-packing. The value of $\tau$ depends on the relative sizes of the colour classes in the optimal colourings of $H$. The proof is algorithmic, which shows that the problem of finding a maximum $H$-packing is polynomially solvable for graphs $G$ whose minimum degree is at least $(\tau+\gamma) n$. On the other hand, given any positive constant $\gamma$, we show that for infinitely many (non-bipartite) graphs $H$ the corresponding decision problem becomes NP-complete if one considers input graphs $G$ of minimum degree at least $(\tau-\gamma) n$.
\end{abstract}

\section{INTRODUCTION}

Given two graphs $H$ and $G$, an $H$-packing in $G$ is a collection of vertex-disjoint copies of $H$ in $G$. $H$-packings are natural generalizations of graph matchings (which correspond to the case when $H$ consists of a single edge). An $H$-packing in $G$ is called perfect if it covers all vertices of $G$. In this case, we also say that $G$ contains an $H$-factor or a perfect $H$-matching. Hell and Kirkpatrick [9] showed that the decision problem whether a graph $G$ has a perfect $H$-packing is NP-complete if and only if $H$ has a component which contains at least 3 vertices. They were motivated by questions arising in timetabling (see [8]).

Hurkens and Schrijver [10] gave an $(|H| / 2+\varepsilon$ )-approximation algorithm (where $\varepsilon>0$ is arbitrary) for the optimization problem of finding a maximum $H$-packing. On the other hand, Kann [11] proved that the problem is APX-hard if $H$ has a component which contains at least three vertices. (In other words, it is impossible to approximate the optimum solution within an arbitrary factor unless $\mathrm{P}=\mathrm{NP}$.) The results in [9] imply that in the remaining cases the problem can be solved in polynomial time. Moreover the following theorem of Alon and Yuster [3] shows that the problem can be solved in polynomial time for instances $G$ which are sufficiently dense:

Theorem 1. [Alon and Yuster [3]] For every $\gamma>0$ and each graph $H$ there exists an integer $n_{0}=n_{0}(\gamma, H)$ such that every graph $G$ whose order $n \geq n_{0}$ is divisible by $|H|$ and whose minimum degree is at least $(1-1 / \chi(H)+\gamma) n$ contains a perfect $H$-packing. Moreover, there is an algorithm which finds this $H$-packing in time $O\left(n^{2.376}\right)$.

Komlós, Sárközy and Szemerédi [17] showed that the term $\gamma n$ in Theorem 1 can be replaced by a constant $C(H)>0$ depending only on $H$, which had been conjectured in [3]. As observed in [3], there are graphs $H$ for which this constant $C(H)$ cannot be omitted completely.

On the other hand, there are graphs $H$ for which the bound on the minimum degree can be improved significantly: for the case where $H=K_{\ell}^{-}$(i.e. a complete graph with one edge removed) and $\ell \geq 4$, we [19] proved that one can replace the chromatic number with the critical chromatic number in Theorem 1. Here the critical chromatic number $\chi_{c r}(H)$ of a graph $H$ is defined as $(\chi(H)-1) h /(h-\sigma(H))$, where $\sigma(H)$ denotes the minimum size of the smallest colour class in a colouring of $H$ with $\chi(H)$ colours and where $h$ denotes the order of $H$. Note that $\chi_{c r}(H)$ always satisfies $\chi(H)-1<\chi_{c r}(H) \leq \chi(H)$ and equals $\chi(H)$ if and only if for every colouring of $H$ with $\chi(H)$ colours, all of the colour classes have equal size. Up to the error term 
$\gamma n$, the minimum degree condition of the result in [19] is best possible. The case $\ell=4$ was solved earlier by Kawarabayashi [12] with the exact bound on the minimum degree.

Here, we determine all non-bipartite graphs $H$ for which the critical chromatic number is the relevant parameter which guarantees a perfect $H$-packing. We also show that for all remaining non-bipartite graphs as well as for all connected bipartite ones, Theorem 1 is best possible up to the error term $\gamma n$.

We say that a colouring of $H$ is optimal if it uses exactly $\chi(H)=: \ell$ colours and call an optimal colouring of $H$ minimal if the smallest colour class has size $\sigma(H)$. Let $\mathcal{C}_{\text {opt }}(H)$ denote the set of all optimal colourings. Given a colouring $c \in \mathcal{C}_{\text {opt }}(H)$, let $x_{1} \leq x_{2} \leq \cdots \leq x_{\ell}$ denote the sizes of the colour classes of $c$. Put $\mathcal{D}(c):=\left\{x_{i+1}-x_{i} \mid i=1, \ldots, \ell-1\right\}$. Let $\mathcal{D}(H)$ denote the union of all the sets $\mathcal{D}(c)$ with $c \in \mathcal{C}_{\text {opt }}(H)$. Write $\operatorname{hcf}(H)$ for the highest common factor of all integers in $\mathcal{D}(H)$. If $\mathcal{D}(H)=\{0\}$ we define $\operatorname{hcf}(H):=1$. Note that $\mathcal{D}(H)=\{0\}$ if and only if $\chi(H)=\chi_{c r}(H)$. Moreover, it is easy to see that there are graphs $H$ with $\operatorname{hcf}(H)=1$ but such that for all colourings $c \in \mathcal{C}_{\text {opt }}(H)$ the highest common factor of all integers in $\mathcal{D}(c)$ is strictly bigger than 1 . Thus for such graphs $H$ there is no single optimal colouring which 'certifies' that $\operatorname{hcf}(H)=1$.

Theorem 2. For every positive constant $\gamma$ and every graph $H$ with $h c f(H)=1$ and $\chi(H) \geq 3$ there exists an integer $n_{0}=n_{0}(\gamma, H)$ such that every graph $G$ whose order $n \geq n_{0}$ is divisible by $|H|$ and whose minimum degree is at least $\left(1-1 / \chi_{c r}(H)+\gamma\right) n$ contains a perfect $H$-packing. Moreover, this perfect $H$-packing can be found in time $O\left(n^{3.376}\right)$.

Theorem 2 is essentially best possible in three ways. Firstly, in Proposition 4 we show that for those non-bipartite graphs $H$ to which Theorem 2 does not apply, Theorem 1 is best possible up to the error term $\gamma n$. This is also easily seen to be the case when $H$ is connected and bipartite (see Section 2). The case when $H$ is bipartite but not connected will be covered in [20].

Secondly, in Proposition 5 we show that for all graphs $H$ a minimum degree of at least $\left(1-1 / \chi_{c r}(H)\right) n$ is necessary to guarantee a perfect $H$-packing. An easy modification of the examples in Proposition 5 shows that there are graphs $H$ for which the term $\gamma n$ in Theorem 2 cannot be replaced by 0 . By using much more involved arguments than those described here, in [20] we show that the term $\gamma n$ can be replaced by a constant depending only on $H$.

Thirdly, the algorithmic assertion of Theorem 2 (and Theorem 1) is also best possible for many graphs $H$. More precisely, suppose that $H$ is either a $K_{\ell}$ with $\ell \geq 3$ or a complete $\ell$-partite graph whose second smallest vertex class has size at least 2. For any such $H$ and any fixed $\gamma>0$ the problem of deciding whether a graph $G$ has a perfect $H$-packing is NP-complete for the class of all instances $G$ with minimum degree at least $\left(1-1 / \chi_{c r}(H)-\gamma\right) n$. It would be very interesting to know whether this hardness result can be extended to all connected graphs $H$ on at least 3 vertices. See Section 3 for details.

Another class of graphs for which $H$-packing problems have been studied are planar graphs. Berman et al. [6] have shown that if $H$ is a connected outerplanar graph and all the instances $G$ are planar the perfect $H$-packing problem is still NP-complete. On the other hand, they showed that the problem can be solved in linear time if $H$ is a planar triangulation on at least 4 vertices. Baker [5] showed that the corresponding maximization problem admits a polynomial time approximation scheme for any planar graph $H$ (i.e. the solution can be approximated within any given constant factor in polynomial time).

In our proof of Theorem 2 we will use the following result of Komlos [14]. It gives an essentially best possible bound on the minimum degree of a graph $G$ which ensures the existence of an almost perfect $H$-packing in $G$. Here the critical chromatic number is the relevant parameter for any graph $H$. 
Theorem 3. [Komlós [14]] For every graph $H$ and every $\gamma_{1}>0$ there exists an integer $n_{1}=$ $n_{1}\left(\gamma_{1}, H\right)$ such that every graph $G$ of order $n \geq n_{1}$ and minimum degree at least $\left(1-1 / \chi_{c r}(H)\right) n$ contains an $H$-packing which covers all but at most $\gamma_{1} n$ vertices of $G$.

The bound on the number of leftover vertices in Theorem 3 was reduced to a constant depending only on $H$ by Shokoufandeh and Zhao [22], but we will not make use of this.

\section{Notation, tools And eXtremal EXAmples}

Throughout this paper we omit floors and ceilings whenever this does not affect the argument. We write $e(G)$ for the number of edges of a graph $G,|G|$ for its order, $\delta(G)$ for its minimum degree, $\Delta(G)$ for its maximum degree, $\chi(G)$ for its chromatic number and $\chi_{c r}(G)$ for its critical chromatic number as defined in Section 1. We denote the degree of a vertex $x \in G$ by $d_{G}(x)$ and its neighbourhood by $N_{G}(x)$.

Given disjoint $A, B \subseteq V(G)$, an $A-B$ edge is an edge of $G$ with one endvertex in $A$ and the other in $B$; the number of these edges is denoted by $e_{G}(A, B)$ or $e(A, B)$ if this is unambiguous. We write $(A, B)_{G}$ for the bipartite subgraph of $G$ whose vertex classes are $A$ and $B$ and whose edges are all $A-B$ edges in $G$. More generally, we write $(A, B)$ for a bipartite graph with vertex classes $A$ and $B$.

The next proposition implies that for those non-bipartite graphs $H$ to which Theorem 2 does not apply Theorem 1 is essentially best possible.

Proposition 4. Let $H$ be a graph such that $\chi(H)>2$ and $h c f(H)>1$. Then there are infinitely many graphs $G$ whose order $n$ is divisible by $|H|$ and whose minimum degree is $(1-1 / \chi(H)) n-1$ but which do not contain a perfect $H$-packing.

Proof. Put $\ell:=\chi(H)$. Given $k \in \mathbb{N}$, let $G$ denote the complete $\ell$-partite graph with vertex classes $U_{1}, \ldots, U_{\ell}$ where $\left|U_{1}\right|=k|H|-1,\left|U_{2}\right|=k|H|+1$ and $\left|U_{i}\right|=|H| k$ for $i=3, \ldots, \ell$. Thus $|G|=k \ell|H|$ and $\delta(G)=(\ell-1) k|H|-1=(1-1 / \chi(H))|G|-1$. Thus it remains to show that $G$ does not have a perfect $H$-packing. So consider disjoint copies $H_{1}, \ldots, H_{j}$ of $H$ in $G$. For all $i=1, \ldots, j$ put $x_{i}:=\left|U_{1} \backslash V\left(H_{1} \cup \cdots \cup H_{i}\right)\right|$ and $y_{i}:=\left|U_{3} \backslash V\left(H_{1} \cup \cdots \cup H_{i}\right)\right|$. By induction it follows that $y_{i}-x_{i} \equiv 1 \bmod \operatorname{hcf}(H)$ for every $i$. As $\operatorname{hcf}(H)>1$ this implies that $x_{j} \neq y_{j}$ and so at least one of $x_{j}, y_{j}$ is nonzero. This shows that no $H$-packing covers all the vertices in $U_{1} \cup U_{3}$ and thus $G$ cannot contain a perfect $H$-packing.

Note that Proposition 4 carries over to connected graphs $H$ of chromatic number 2. Indeed, let $k \in \mathbb{N}$ be odd and let $G$ be the disjoint union of two cliques of order $\lfloor|H| k / 2\rfloor$ and $\lceil|H| k / 2\rceil$. Then the minimum degree of $G$ is $\lfloor(1-1 / \chi(H))|G|\rfloor-1$. However it is easy to check that $\lfloor|H| k / 2\rfloor$ is not divisible by $|H|$. Thus $G$ cannot contain a perfect $H$-packing as $H$ is connected.

For completeness we also include the short proof of the fact that a minimum degree of at least $\left(1-1 / \chi_{c r}(H)\right)|G|$ is necessary to guarantee a perfect $H$-packing in a graph $G$. This fact was already observed in [14].

Proposition 5. For every graph $H$ with $\chi(H) \geq 2$ there are infinitely many graphs $G$ whose order $n$ is divisible by $|H|$ and whose minimum degree is $\left(1-1 / \chi_{c r}(H)\right) n-1$ but which do not contain a perfect $H$-packing.

Proof. Let $\ell:=\chi(H)$. Consider any minimal colouring of $H$ and let $x_{1} \leq \cdots \leq x_{\ell}$ denote the sizes of the colour classes. Thus $x_{1}=\sigma(H)$. Let $s_{1}:=(\ell-1) x_{1}$ and $s:=x_{2}+\cdots+x_{\ell}$. Given $k \in \mathbb{N}$, let $G$ denote the complete $\ell$-partite graph with vertex classes $U_{1}, \ldots, U_{\ell}$ where $\left|U_{1}\right|=k s_{1}-1,\left|U_{2}\right|=k s+1$ and $\left|U_{i}\right|=k s$ for every $i \geq 3$. Then $|G|=k(\ell-1)|H|$ and $\delta(G)=(\ell-2) k s+k s_{1}-1=\left(1-1 / \chi_{c r}(H)\right)|G|-1$. However, every copy of $H$ in $G$ contains at most $|H|-x_{1}=s$ vertices in $U_{2} \cup \cdots \cup U_{\ell}$. Thus any $H$-packing in $G$ covers at most $s\left|U_{1}\right| / x_{1}<|G|-\left|U_{1}\right|$ vertices in $U_{2} \cup \cdots \cup U_{\ell}$. So $G$ cannot contain a perfect $H$-packing. 
Given a graph $H$ as in Theorem 2, the following lemma gives a sufficient condition on the sizes of the vertex classes of a complete $\chi(H)$-partite graph $G$ which ensures that $G$ has a perfect $H$-packing. Its proof will appear in [20].

Lemma 6. Let $H$ be a graph with $\ell:=\chi(H) \geq 3, \chi_{c r}(H)<\chi(H)$ and hcf $(H)=1$. Put $\xi:=(\ell-1) \sigma(H) /(|H|-\sigma(H))$. There exists a positive constant $\beta_{0}=\beta_{0}(H) \leq \xi^{4}$ such that for every positive $\beta \leq \beta_{0}$ there exists an integer $u_{0}=u_{0}(H, \beta)$ for which the following holds. Let $\eta$ be a positive constant with $\eta^{4} \geq \beta$ and $\eta \leq 1-\xi$. Suppose that $G$ is a complete $\ell$-partite graph with vertex classes $U_{1}, \ldots, U_{\ell}$ such that $|H|$ divides $|G|$. Let $u_{i}:=\left|U_{i}\right|$ for all $i$. Suppose that $u_{0} \leq u_{2} \leq \cdots \leq u_{\ell} \leq(1+\beta) u_{2}$ and $u_{1}=(1+\eta) \xi u_{2}$. Then $G$ contains a perfect $H$-packing.

\section{HARDNESS RESUlts FOR ALGORITHMS}

For convenience, let $\operatorname{Pack}(H)$ denote the problem of deciding whether a graph $G$ contains a perfect $H$-packing. We also need to define a restricted version of this problem. For this, we say that a graph $G$ is $c$-dense if it has minimum degree at least $c|G|$. Let $\operatorname{Pack}(H, c)$ denote the problem of deciding whether a $c$-dense input graph $G$ contains a perfect $H$-packing.

Theorem 7. Let $\ell \geq 2$ be an integer. Let $H$ be a graph such that either $H=K_{\ell}$ and $\ell \geq 3$ or such that $H$ is a complete $\ell$-partite graph with vertex classes of size $x_{1} \leq \cdots \leq x_{\ell}$ where $x_{2} \geq 2$. Let $\gamma$ be such that $0<\gamma<1-1 / \chi_{c r}(H)$. Then $\operatorname{Pack}(H, c)$ is NP-complete for any $c \leq 1-1 / \chi_{c r}(H)-\gamma$.

Proof. We first consider the case when $H$ is a complete $\ell$-partite graph with vertex classes of size $x_{1} \leq \cdots \leq x_{\ell}$ where $x_{2} \geq 2$. Let $H^{\prime}:=K_{x_{1}, x_{2}}$ and fix $c \leq 1-1 / \chi_{c r}(H)-\gamma$. We will prove the result by a reduction from the $H^{\prime}$-packing problem. Since $H^{\prime}$ is a connected graph on at least 3 vertices, the latter problem was proved to be NP-complete by Hell and Kirkpatrick [8]. Thus, it suffices to show that for every instance $G$ of $\operatorname{Pack}\left(H^{\prime}\right)$, we can construct (in polynomial time) an instance $F=F(G)$ of $\operatorname{Pack}(H, c)$, so that $F$ has a perfect $H$-packing if and only if $G$ has a perfect $H^{\prime}$-packing.

Let $z_{1}:=(\ell-1) x_{1}$ and $z:=x_{2}+\cdots+x_{\ell}$. Let $B^{*}$ denote the complete $\ell$-partite graph with one vertex class of size $z_{1}$ and $\ell-1$ vertex classes of size $z$. Given an integer $t$, let $B^{*}(t)$ denote the complete $\ell$-partite graph with one vertex class $Z_{1}$ of size $z_{1} t$ and $\ell-1$ vertex classes $Z_{2}, \ldots, Z_{\ell}$ of size $z t$. Note that $B^{*}(t)$ has a perfect $H$-packing consisting of $(\ell-1) t$ copies of $H$.

Let $t:=\lceil|G| / \gamma\rceil$ and $k:=|G| /\left|H^{\prime}\right|$. Clearly, we may assume that $k$ is an integer. We now obtain $F$ from the disjoint union of $B^{*}(t), G$ and $\ell-2$ independent sets $I_{3}, \ldots, I_{\ell}$ of sizes $k x_{3}, \ldots, k x_{\ell}$ by connecting all vertices of $G$ to all vertices of $B^{*}(t)-Z_{2}$ as well as to all vertices in $I_{3} \cup \cdots \cup I_{\ell}$. Moreover, for all $i=3, \ldots, \ell$, we connect all vertices in $I_{i}$ to all vertices of $B^{*}(t)-Z_{i}$ as well as to all vertices in each $I_{j}$ with $j \neq i$. It is easily seen that $F$ is $c$-dense.

Clearly, if $G$ has a perfect $H^{\prime}$-packing, then $F$ has a perfect $H$-packing. Now suppose that $G$ does not have a perfect $H^{\prime}$-packing. We have to show that $F$ does not have a perfect $H$-packing. So suppose that $\mathcal{H}$ is a perfect $H$-packing in $F$. Given $0 \leq j<x_{1}$, let $\mathcal{H}_{j}(G) \subseteq \mathcal{H}$ denote the collection of all those copies of $H$ which do not avoid $G$ and meet $Z_{1}$ in precisely $j$ vertices. Note that each such copy meets $G$ in at least $x_{1}+x_{2}-j$ vertices and it avoids $Z_{2}$. Put $n_{j}=\left|\mathcal{H}_{j}(G)\right|$. So there exists an integer $D \geq 0$ such that

$$
D+\sum_{j=0}^{x_{1}-1} n_{j}\left(x_{1}+x_{2}-j\right)=|G|=\left(x_{1}+x_{2}\right) k .
$$

Moreover, if a copy of $H$ in $\mathcal{H}_{0}(G)$ meets $G$ in precisely $x_{1}+x_{2}$ vertices, then these vertices form the $H^{\prime}$-subgraph of that copy. Since $G$ does not have a perfect $H^{\prime}$-packing, it follows that either $D>0$ or $n_{j}>0$ for some $j \geq 1$ (or both). Define $D^{\prime}$ by setting $D^{\prime}:=D$ if $D>0$ and 
$D^{\prime}:=-1$ if $D=0$. Thus (1) implies that

$$
\sum_{j=0}^{x_{1}-1} n_{j} \geq k-D^{\prime} /\left(x_{1}+x_{2}\right) .
$$

Let $\mathcal{H}(G)$ denote the union of all the $\mathcal{H}_{j}(G)$. Since every copy of $H$ in $\mathcal{H} \backslash \mathcal{H}(G)$ contains at least $x_{1}$ vertices in $Z_{1}$ there are at most $\left(\left|Z_{1}\right|-\sum_{j=0}^{x_{1}-1} j n_{j}\right) / x_{1}$ such copies. Since all these copies have to cover all those vertices in $F-Z_{1}$ which do not belong to copies of $H$ in $\mathcal{H}(G)$ it follows that

$$
\frac{t z_{1}-\sum_{j=0}^{x_{1}-1} j n_{j}}{x_{1}} \cdot z \geq\left|F-Z_{1}\right|-\sum_{j=0}^{x_{1}-1} n_{j}(|H|-j)=(\ell-1) t z+k|H|-\sum_{j=0}^{x_{1}-1} n_{j}(|H|-j) .
$$

This in turn implies that $\sum_{j=0}^{x_{1}-1} n_{j}\left(x_{1}-j\right) \geq x_{1} k$. Together with (1) the latter implies that $\sum_{j=0}^{x_{1}-1} n_{j} \leq k-D / x_{2}$. However, this contradicts (2) and thus completes the proof that $F$ does not have a perfect $H$-packing.

Now consider the case when $H=K_{\ell}$. This time, we will prove the result by a reduction from the $H$-packing problem. Thus for every instance $G$ of $\operatorname{Pack}(H)$, we will construct an instance $F=F(G)$ of $\operatorname{Pack}(H, c)$, so that $F$ has a perfect $H$-packing if and only if $G$ has one. This time, $F$ is obtained from the complete $\ell$-partite graph with vertex classes $Z_{1}, \ldots, Z_{\ell}$ of size $t:=\lceil|G| / \gamma\rceil$ by adding $G$ and connecting all vertices of $G$ to all vertices in $Z_{1} \cup \cdots \cup Z_{\ell-1}$. Again, it is easily seen that $F$ is $c$-dense.

Clearly, if $G$ has a perfect $H$-packing, then $F$ has one too. Now suppose that $G$ does not have a perfect $H$-packing but that $\mathcal{H}$ is a perfect $H$-packing in $F$. Let $\mathcal{H}(G) \subseteq \mathcal{H}$ denote the collection of all those copies of $H$ which meet $G$. Every such copy of $H$ avoids $Z_{\ell}$. Moreover, since $G$ does not have a perfect $H$-packing, at least one vertex of $Z_{1} \cup \cdots \cup Z_{\ell-1}$ is contained in some copy of $H$ in $\mathcal{H}(G)$. Consider the subgraph $F^{\prime}$ of $F$ obtained by deleting the vertices in all the copies of $H$ in $\mathcal{H}(G)$. Then the $\ell$ th vertex class of $F^{\prime}$ still has size $\left|Z_{\ell}\right|$ whereas the union of all the other vertex classes of $F^{\prime}$ has size $<(\ell-1)\left|Z_{\ell}\right|$. Thus $F^{\prime}$ does not contain a perfect $H$-packing, a contradiction.

Note that the graph $F$ constructed in the reduction has size polynomial in $|G|$ even if $\gamma=$ $|G|^{-K}$, say, where $K>0$ is an arbitrary constant. Thus, given any constant $K>0$, the assertion of the theorem actually remains valid for the class of all instances $G$ whose minimum degree is at least $\left(1-1 / \chi_{c r}(H)-|G|^{-K}\right)|G|$.

It would be interesting to know whether the assertion of the theorem can be extended to all graphs $H$ for which $\operatorname{Pack}(H)$ is NP-complete (i.e. those which contain a component with at least 3 vertices). Similarly, it might be true that the optimization problem of finding a maximum $H$-packing is APX-complete for all such graphs $H$ and for all instances $G$ whose minimum degree is at least $\left(1-1 / \chi_{c r}(H)-\gamma\right)|G|$. This is open even if $H$ is a triangle.

\section{The Regularity lemma and the Blow-up lemma}

The purpose of this section is to collect all the information we need about Szemerédi's Regularity lemma and and the Blow-up lemma of Komlós, Sárközy and Szemerédi [15]. See [18] and [13] for surveys about these. Let us start with some more notation. The density of a bipartite graph $G=(A, B)$ is defined to be $d(A, B):=\frac{e(A, B)}{|A||B|}$. Given $\varepsilon>0$, we say that $G$ is $\varepsilon$-regular if for all sets $X \subseteq A$ and $Y \subseteq B$ with $|X| \geq \varepsilon|A|$ and $|Y| \geq \varepsilon|B|$ we have $|d(A, B)-d(X, Y)|<\varepsilon$. Given $d \in[0,1]$, we say that $G$ is $(\varepsilon, d)$-superregular if all sets $X \subseteq A$ and $Y \subseteq B$ with $|X| \geq \varepsilon|A|$ and $|Y| \geq \varepsilon|B|$ satisfy $d(X, Y)>d$ and, furthermore, if $d_{G}(a)>d|B|$ for all $a \in A$ and $d_{G}(b)>d|A|$ for all $b \in B$. 
We will use the following degree form of Szemerédi's Regularity lemma which can be easily derived from the classical version. Proofs of the latter are for example included in [4] and [7].

Lemma 8 (Regularity lemma). For all $\varepsilon>0$ and all integers $k_{0}$ there is an $N=N\left(\varepsilon, k_{0}\right)$ such that for every number $d \in[0,1]$ and for every graph $G$ on at least $N$ vertices there exist a partition of $V(G)$ into $V_{0}, V_{1}, \ldots, V_{k}$ and a spanning subgraph $G^{\prime}$ of $G$ such that:

- $k_{0} \leq k \leq N,\left|V_{0}\right| \leq \varepsilon|G|,\left|V_{1}\right|=\cdots=\left|V_{k}\right|=: L$,

- $d_{G^{\prime}}(x)>d_{G}(x)-(d+\varepsilon)|G|$ for all vertices $x \in G$,

- for all $i \geq 1$ the graph $G^{\prime}\left[V_{i}\right]$ is empty and for all $1 \leq i<j \leq k$ the graph $\left(V_{i}, V_{j}\right)_{G^{\prime}}$ is $\varepsilon$-regular and has density either 0 or $>d$.

The sets $V_{i}(i \geq 1)$ are called clusters, $V_{0}$ is called the exceptional set. Given clusters and $G^{\prime}$ as in Lemma 8, the reduced graph $R$ is the graph whose vertices are $V_{1}, \ldots, V_{k}$ and in which $V_{i}$ is joined to $V_{j}$ whenever $\left(V_{i}, V_{j}\right)_{G^{\prime}}$ is $\varepsilon$-regular and has density $>d$. Thus $V_{i} V_{j}$ is an edge of $R$ if and only if $G^{\prime}$ has an edge between $V_{i}$ and $V_{j}$.

We will also use the Blow-up lemma of Komlós, Sárközy and Szemerédi [15]. It implies that dense regular pairs behave like complete bipartite graphs with respect to containing bounded degree graphs as subgraphs.

Lemma 9 (Blow-up lemma). Given a graph $F$ on $\{1, \ldots, f\}$ and numbers $d, \Delta>0$, there is a positive number $\varepsilon_{0}=\varepsilon_{0}(d, \Delta, f)$ such that the following holds. Given $L_{1}, \ldots, L_{f} \in \mathbb{N}$ and $\varepsilon \leq \varepsilon_{0}$, let $F^{*}$ be the graph obtained from $F$ by replacing each vertex $i \in F$ with a set $V_{i}$ of $L_{i}$ new vertices and joining all vertices in $V_{i}$ to all vertices in $V_{j}$ whenever ij is an edge of $F$. Let $G$ be a spanning subgraph of $F^{*}$ such that for every edge ij $\in F$ the graph $\left(V_{i}, V_{j}\right)_{G}$ is $(\varepsilon, d)$-superregular. Then $G$ contains a copy of every subgraph $H$ of $F^{*}$ with $\Delta(H) \leq \Delta$.

\section{Proof of Theorem 2}

5.1. Further notation and sketch of the proof. Before we can outline the idea of the proof, we need to introduce some more notation. Let $H, G$ and $\gamma$ be as in Theorem 2. By Theorem 1 we may assume that $\chi_{c r}(H)<\chi(H)$. Put $\ell:=\chi(H) \geq 3$. Fix a minimal colouring $c \in \mathcal{C}_{\text {opt }}(H)$ and let $x_{1} \leq x_{2} \leq \cdots \leq x_{\ell}$ denote sizes of the colour classes of $c$. So $\sigma(H)=x_{1}$. Put $z_{1}:=(\ell-1) x_{1}, z:=x_{2}+\cdots+x_{\ell}$ and

$$
\xi:=\frac{z_{1}}{z}=\frac{(\ell-1) \sigma(H)}{|H|-\sigma(H)} .
$$

Let $B^{*}$ denote the complete $\ell$-partite graph with one vertex class of size $z_{1}$ and $\ell-1$ vertex classes of size $z$. Note that $B^{*}$ has a perfect $H$-packing consisting of $\ell-1$ copies of $H$. Moreover,

$$
\chi_{c r}(H)=\chi_{c r}\left(B^{*}\right)=\ell-1+\xi .
$$

Let $s$ be a sufficiently large integer and $\lambda \ll \gamma, \xi, 1-\xi$ be a positive constant. Let $B^{\prime}$ denote the complete $\ell$-partite graph with one vertex class of size $s_{1}:=\xi(1+\lambda) s$ and $\ell-1$ vertex classes of size $s$. Moreover, we choose $\lambda$ and $s$ in such a way that $B^{\prime}$ contains a perfect $B^{*}$-packing. Note that

$$
\chi_{c r}\left(B^{\prime}\right)=\frac{(\ell-1)\left|B^{\prime}\right|}{\left|B^{\prime}\right|-s_{1}}=\ell-1+\xi(1+\lambda) .
$$

We now give an outline of the proof of Theorem 2. We first apply the Regularity lemma to our given graph $G$ in order to obtain a reduced graph $R$. Then we apply Theorem 3 to $R$ to obtain an almost perfect $B^{\prime}$-packing of $R$. The surplus $\gamma n$ in the minimum degree of $G$ ensures that we are able to apply Theorem 3 here. Next we add all the clusters of $R$ which are not covered by this $B^{\prime}$-packing to the exceptional set $V_{0}$. For each exceptional vertex $x \in V_{0}$ we then choose a copy of $H$ which consists of $x$ together with $|H|-1$ vertices lying in some clusters. All these 
copies of $H$ will be disjoint for distinct exceptional vertices. We remove all the vertices of $G$ contained in these copies from the clusters they belong to.

Our next aim is to apply the Blow-up lemma to each copy $B_{i}^{\prime}$ of $B^{\prime}$ in the $B^{\prime}$-packing of $R$ in order to find an $H$-packing in $G$ which covers all the vertices belonging to (the modified) clusters in $B_{i}^{\prime}$. Then all these $H$-packings together with all the copies of $H$ chosen so far for the exceptional vertices would form a perfect $H$-packing in $G$. However, a necessary condition for this is that the complete $\ell$-partite graph $F_{i}^{*}$ whose $j$ th vertex class is the union of all clusters in the $j$ th vertex class of $B_{i}^{\prime}$ (for all $j=1, \ldots, \ell$ ) has a perfect $H$-packing. We will apply Lemma 6 to ensure this. One condition in Lemma 6 is that $|H|$ has to divide $\left|F_{i}^{*}\right|$. It turns out that this can be achieved by taking out a bounded number of suitable further copies of $H$. The second condition in Lemma 6 is that the $\ell-1$ larger vertex classes of $F_{i}^{*}$ must have roughly the same size, $u$ say, while the smallest vertex should be a little larger than $\xi u$. But this second condition was the reason why we chose a $B^{\prime}$-packing in $R$ and not simply a $B^{*}$-packing: the ratio of the size of the smallest vertex class of $B^{\prime}$ to the size of the other classes is $\xi(1+\gamma)$ and in subsequent steps of the proof, we changed this ratio only by a comparatively small amount, so we can still satisfy the second condition Lemma 6 with room to spare.

\subsection{Applying the Regularity lemma and choosing the packing in the reduced graph.}

We will fix further constants satisfying the following hierarchy

$$
0<\varepsilon \ll d \ll \gamma_{1} \ll \beta \ll \alpha \ll \lambda \ll \gamma, \xi, 1-\xi .
$$

Moreover, we choose $\beta, \gamma_{1}$ and a new integer $k_{0}$ such that

$$
\beta \ll \beta_{0}(H), \quad \gamma_{1} \ll 1 /\left|B^{\prime}\right|, \quad k_{0} \geq n_{1}\left(\gamma_{1}, B^{\prime}\right),
$$

where $\beta_{0}(H)$ is as defined in Lemma 6 and $n_{1}$ is as defined in Theorem 3. In what follows, we assume that the order $n$ of our given graph $G$ is sufficiently large for our estimates to hold. We now apply the Regularity lemma with parameters $\varepsilon$ and $d$ to our given graph $G$ to obtain clusters, an exceptional set $V_{0}$ and a reduced graph $R$. (3) and (5) together with the wellknown fact that the minimum degree of $G$ is almost inherited by its reduced graph (see e.g. [21, Prop. 9]) imply that

$$
\delta(R) \geq\left(1-\frac{1}{\ell-1+\xi}+\frac{\gamma}{2}\right)|R| .
$$

Together with (4) and the fact that $\lambda \ll \gamma$ by (5) this implies that $\delta(R) \geq\left(1-1 / \chi_{c r}\left(B^{\prime}\right)\right)|R|$. Since also $|R| \geq k_{0} \geq n_{1}\left(\gamma_{1}, B^{\prime}\right)$ by (6), we may apply Theorem 3 to $R$ to find a $B^{\prime}$-packing which covers all but at most $\gamma_{1}|R|$ vertices of $R$. We denote the copies of $B^{\prime}$ in this packing by $B_{1}^{\prime}, \ldots, B_{k^{\prime}}^{\prime}$. We delete all the clusters not contained in some $B_{i}^{\prime}$ from $R$ and add all the vertices lying in these clusters to the exceptional set $V_{0}$. Thus $\left|V_{0}\right| \leq \varepsilon n+\gamma_{1} n \leq 2 \gamma_{1} n$. From now on, we denote by $R$ the subgraph of the reduced graph induced by all the remaining clusters. Since $\gamma_{1} \ll \gamma$ we still have that

$$
\delta(R) \geq\left(1-\frac{1}{\ell-1+\xi}+\frac{\gamma}{4}\right)|R| .
$$

Recall that by definition of $B^{\prime}$, each $B_{i}^{\prime}$ contains a perfect $B^{*}$-packing. Fix such a $B^{*}$-packing for each $i=1, \ldots, k^{\prime}$. The union of all these $B^{*}$-packings gives us a perfect $B^{*}$-packing $\mathcal{B}^{*}$ in $R$. (Instead of introducing $\mathcal{B}^{*}$, we could also have worked with the $B^{\prime}$-packing throughout, but considering $\mathcal{B}^{*}$ does simplify some calculations later on.)

Let $L^{\prime}:=\left(1-\varepsilon\left|B^{\prime}\right|\right) L$. It is easy to check that for all $i=1, \ldots, k^{\prime}$ we can replace each cluster $V_{a}$ in $B_{i}^{\prime}$ by a subcluster of size $L^{\prime}$ such that for each edge $V_{a} V_{b}$ of $B_{i}^{\prime}$ the bipartite subgraph of $G^{\prime}$ between the chosen subclusters of $V_{a}$ and $V_{b}$ is $(2 \varepsilon, d / 2)$-superregular (see e.g. [21, Prop. 8]). 
Add all the vertices of $G$ which do not lie in one of the chosen subclusters to the exceptional set $V_{0}$. Then

$$
\left|V_{0}\right| \leq 3 \gamma_{1} n
$$

From now on, we refer to the chosen subclusters as the clusters of $R$. Next we partition each of these clusters $V_{a}$ into a red part $V_{a}^{\text {red }}$ and a blue part $V_{a}^{\text {blue }}$ such that ||$V_{a}^{\text {red }}|-| V_{a}^{\text {blue }}|| \leq \varepsilon L^{\prime}=$ $\varepsilon\left|V_{a}\right|$ and such that ||$N_{G}(x) \cap V_{a}^{\text {red }}|-| N_{G}(x) \cap V_{a}^{\text {blue }}|| \leq \varepsilon L^{\prime}$ for every vertex $x \in G$. (Consider a random partition to see that there are $V_{a}^{\text {red }}$ and $V_{a}^{\text {blue }}$ with these properties.) Together all these partitions of the clusters of $R$ yield a partition of the vertices of $G-V_{0}$ into red and blue vertices. We will use these partitions to ensure that even after some modifications which we have to carry out during the proof, the edges of the $B_{i}^{\prime}$ will still correspond to superregular subgraphs of $G^{\prime}$. More precisely, in Section 5.3 we will choose certain copies of $H$ in $G$, but each copy will avoid all the red vertices. All the vertices contained in these copies of $H$ will be removed from the clusters they belong to. However, if we look at the (modified) bipartite subgraph of $G^{\prime}$ which corresponds to some edge $V_{a} V_{b}$ of $B_{i}^{\prime}$, then this subgraph of $G^{\prime}$ will still be $(5 \varepsilon, d / 5)$-superregular since it still contains all vertices in $V_{a}^{r e d}$ and $V_{b}^{r e d}$. After Section 5.3, we will remove only a bounded number of further vertices from the clusters, which will also not affect the superregularity significantly.

5.3. Incorporating the exceptional vertices. Given an exceptional vertex $x \in V_{0}$, we call a copy $B \in \mathcal{B}^{*}$ of $B^{*}$ useful for $x$ if there are $\ell-1$ clusters belonging to different vertex classes of $B$ such that $x$ has at least $\alpha L^{\prime}$ neighbours in each of these clusters. Let $k_{x}$ denote the number of copies of $B^{*}$ in $\mathcal{B}^{*}$ which are useful for $x$. Then

$$
\begin{aligned}
k_{x} L^{\prime}\left|B^{*}\right|+\left(\left|\mathcal{B}^{*}\right|-k_{x}\right)\left(\left|B^{*}\right| L^{\prime}-(1-\alpha) L^{\prime}\left(z_{1}+z\right)\right) & \geq d_{G}(x)-\left|V_{0}\right| \\
& \stackrel{(3),(5),(8)}{\geq}\left(1-\frac{1}{\ell-1+\xi}+\frac{\gamma}{2}\right) L^{\prime}\left|B^{*}\right|\left|\mathcal{B}^{*}\right| .
\end{aligned}
$$

A straightforward calculation now shows that $k_{x} \geq \frac{\xi\left|\mathcal{B}^{*}\right|}{2}$. Since $\gamma_{1} \ll \beta, \xi, 1 /\left|B^{*}\right|$ by (5) and (6), this in turn shows that $k_{x} \beta L^{\prime} \gg\left|V_{0}\right|$. Thus we can assign each exceptional vertex $x \in V_{0}$ greedily to some $B_{x} \in \mathcal{B}^{*}$ in such a way that $B_{x}$ is useful for $x$ and that to each $B \in \mathcal{B}^{*}$ we assign at most $\beta L^{\prime}$ exceptional vertices. For each $x \in V_{0}$ we now choose a copy of $H$ in $G$ which contains $x$ and whose other vertices all lie in clusters belonging to $B_{x}$. Since at most $\beta L^{\prime} \ll \alpha L^{\prime}$ exceptional vertices are assigned to $B_{x}$ but $x$ has at least $\alpha L^{\prime}$ neighbours in $\ell-1$ clusters belonging to different vertex classes of $B_{x}$, this can be done in such a way that these copies of $H$ in $G$ are disjoint for distinct exceptional vertices and such that they avoid all the red vertices of $G$. (Indeed, the existence of such copies of $H$ in $G$ easily follows from a 'greedy' argument based on the $\varepsilon$-regularity of the bipartite subgraphs of $G^{\prime}$ corresponding to the edges of the $B_{x} \subseteq R$, see e.g. Lemma 7.3.2 in [7] or Theorem 2.1 in [18]. We will often use this and similar facts below.) We remove all the vertices lying in these copies of $H$ from the clusters they belong to.

5.4. Making the blow-up of each $B \in \mathcal{B}^{*}$ divisible by $|H|$. Given a subgraph $S \subseteq R$, we denote by $V_{G}(S) \subseteq V(G)$ the union of all the clusters belonging to $S$. Our aim is to apply the Blow-up lemma to each copy $B_{i}^{\prime}$ in the $B^{\prime}$-packing of $R$ in order to find a $H$-packing in $G$ which covers all the vertices of $V_{G}\left(B_{i}^{\prime}\right)$. Then all these $H$-packings together with all the copies of $H$ chosen for the exceptional vertices in Section 5.3 yield a perfect $H$-packing in $G$. However, to be able to apply the Blow-up lemma we have to ensure that the complete $\ell$-partite graph whose $j$ th vertex class is the union of all the clusters lying in the $j$ th vertex class of $B_{i}^{\prime}(j=1, \ldots, \ell)$ contains a perfect $H$-packing. By Lemma 6 this will turn out to be the case if $|H|$ divides the order of this complete $\ell$-partite graph, i.e. if $|H|$ divides $\left|V_{G}\left(B_{i}^{\prime}\right)\right|$. To achieve the latter, we will take out a bounded number of further copies of $H$ in $G$ (i.e. we will remove all the vertices lying 
in these copies from the clusters they belong to). In fact, we will take out a bounded number of copies of $H$ in $G$ to achieve that $|H|$ even divides $\left|V_{G}(B)\right|$ for each $B \in \mathcal{B}^{*}$.

For this, we define an auxiliary graph $F$ whose vertices are the elements of $\mathcal{B}^{*}$ and in which $B_{1}, B_{2} \in \mathcal{B}^{*}$ are adjacent if the reduced graph $R$ contains a copy of $K_{\ell}$ with one vertex in $B_{1}$ and $\ell-1$ vertices in $B_{2}$ or vice versa.

To motivate the definition of $F$, let us first consider the case when $F$ is connected. If $B_{1}, B_{2} \in \mathcal{B}^{*}$ are adjacent in $F$ then $G$ contains a copy of $H$ with one vertex in $V_{G}\left(B_{1}\right)$ and all the other vertices in $V_{G}\left(B_{2}\right)$ or vice versa. In fact, we can even find $|H|-1$ disjoint such copies of $H$ in $G$ by using the 'greedy' argument referred to in Section 5.3. Taking out a suitable number of such copies (at most $|H|-1$ ), we can achieve that the size of the subset of $V_{G}\left(B_{1}\right)$ obtained in this way is divisible by $|H|$. Thus we can 'shift the remainders mod $|H|$ ' along a spanning tree of $F$ to achieve that $\left|V_{G}(B)\right|$ is divisible by $|H|$ for each $B \in \mathcal{B}^{*}$. (To see this, use that $\sum_{B \in \mathcal{B}^{*}}\left|V_{G}(B)\right|$ is divisible by $|H|$ since $|G|$ is divisible by $|H|$.)

Thus in what follows we may assume that $F$ is not connected. Let $\mathcal{C}$ denote the set of all components of $F$. Given a component $C$ of $F$, we denote by $V_{R}(C) \subseteq V(R)$ the set of all those clusters which belong to some $B \in \mathcal{B}^{*}$ with $B \in C$. We write $V_{G}(C) \subseteq V(G)$ for the union of all the clusters in $V_{R}(C)$. We first show that we can take out a bounded number of copies of $H$ in $G$ in order to make $\left|V_{G}(C)\right|$ divisible by $|H|$ for each $C \in \mathcal{C}$. After that, we can "shift the remainders mod $|H|$ ' within each component $C \in \mathcal{C}$ along a spanning tree as indicated above to make $\left|V_{G}(B)\right|$ divisible by $|H|$ for each $B \in \mathcal{B}^{*}$. For our argument, we will need the following claim.

Claim 1. Let $C_{1}, C_{2} \in \mathcal{C}$ and let $a \in V_{R}\left(C_{2}\right)$. Then

$$
\left|N_{R}(a) \cap V_{R}\left(C_{1}\right)\right|<\left(1-\frac{1}{\ell-1+\xi}+\frac{\gamma}{4}\right)\left|V_{R}\left(C_{1}\right)\right| .
$$

Suppose not. Then there is some $B \in \mathcal{B}^{*}$ such that $B \in C_{1}$ and such that

$$
\left|N_{R}(a) \cap B\right| \geq\left(1-\frac{1}{\ell-1+\xi}+\frac{\gamma}{4}\right)|B|=|B|-z+\frac{\gamma|B|}{4} .
$$

This implies that $a$ has a neighbour in at least $\ell-1$ vertex classes of $B$. Thus $R$ contains a copy of $K_{\ell}$ which consists of $a$ together with $\ell-1$ of its neighbours in $B$. But by definition of the auxiliary graph $F$, this means that $B$ is adjacent in $F$ to the copy $B_{0} \in \mathcal{B}^{*}$ that contains $a$, i.e. $B$ and $B_{0}$ lie in the same component of $F$, a contradiction. This completes the proof of Claim 1.

Claim 2. There exist a component $C^{\prime} \in \mathcal{C}$, a copy $K$ of $K_{\ell}$ in $R$ and a vertex $a_{0} \in V(R) \backslash$ $\left(V(K) \cup V_{R}\left(C^{\prime}\right)\right)$ such that $K$ meets $V_{R}\left(C^{\prime}\right)$ in exactly one vertex and such that $a_{0}$ is joined to all the remaining vertices in $K$.

As $\delta(R)>1 / 2$, there exists an edge $a_{1} a_{2} \in R$ which joins the vertex sets corresponding to two different components of $F$, i.e. there are distinct $C_{1}, C_{2} \in \mathcal{C}$ such that $a_{1} \in V_{R}\left(C_{1}\right)$ and $a_{2} \in V_{R}\left(C_{2}\right)$. By (7) the number of common neighbours of $a_{1}$ and $a_{2}$ in $R$ is at least

$$
\left(1-\frac{2}{\ell-1+\xi}+\frac{\gamma}{4}\right)|R| \text {. }
$$

To prove the claim, we will now distinguish two cases.

Case 1. At least $\left(1-\frac{2}{\ell-1+\xi}+\frac{\gamma}{4}\right)\left|V(R) \backslash V_{R}\left(C_{1}\right)\right|$ common neighbours of $a_{1}$ and $a_{2}$ lie outside $V_{R}\left(C_{1}\right)$. 
Let $a_{3}$ be a common neighbour of $a_{1}$ and $a_{2}$ outside $V_{R}\left(C_{1}\right)$. Claim 1 and (7) together imply that the number of common neighbours of $a_{1}, a_{2}$ and $a_{3}$ outside $V_{R}\left(C_{1}\right)$ is at least

$$
\left(1-\frac{3}{\ell-1+\xi}+\frac{\gamma}{4}\right)\left|V(R) \backslash V_{R}\left(C_{1}\right)\right| \text {. }
$$

Choose such a common neighbour $a_{4}$. Continuing in this way we obtain distinct vertices $a_{2}, \ldots, a_{\ell}$ outside $V_{R}\left(C_{1}\right)$ which together with $a_{1}$ from a copy $K$ of $K_{\ell}$ in $R$. As before, Claim 1 and (7) together imply that the number of common neighbours of $a_{2}, \ldots, a_{\ell}$ outside $V_{R}\left(C_{1}\right)$ is at least $\gamma\left|V(R) \backslash V_{R}\left(C_{1}\right)\right| / 4$. Let $a_{0}$ be such a common neighbour. Then Claim 2 holds with $C^{\prime}:=C_{1}, K$ and $a_{0}$. Thus we may now consider

Case 2. At least $\left(1-\frac{2}{\ell-1+\xi}+\frac{\gamma}{4}\right)\left|V_{R}\left(C_{1}\right)\right|$ common neighbours of $a_{1}$ and $a_{2}$ lie in $V_{R}\left(C_{1}\right)$.

In this case we proceed similarly as in Case 1 . However, this time we choose $a_{0}, a_{3}, \ldots, a_{\ell}$ inside $V_{R}\left(C_{1}\right)$. Indeed, this can be done since Claim 1 and (7) together imply that each vertex in $V_{R}\left(C_{1}\right)$ has at least $\left(1-\frac{1}{\ell-1+\xi}+\frac{\gamma}{4}\right)\left|V_{R}\left(C_{1}\right)\right|$ neighbours in $V_{R}\left(C_{1}\right)$. Then Claim 2 holds with $C^{\prime}:=C_{2}$.

Claim 3. We can make $\left|V_{G}(B)\right|$ divisible by $|H|$ for all $B \in \mathcal{B}^{*}$ by taking out at most $\left|\mathcal{B}^{*}\right||H|$ copies of $H$ in $G$.

We first take out some copies of $H$ in $G$ to achieve that $\left|V_{G}(C)\right|$ is divisible by $|H|$ for each $C \in \mathcal{C}$. To do this we proceed as follows. We apply Claim 2 to find a component $C_{1} \in \mathcal{C}$, a copy $K$ of $K_{\ell}$ in $R$ and a vertex $a_{0} \in V(R) \backslash\left(V(K) \cup V_{R}\left(C_{1}\right)\right)$ such that $K$ meets $V_{R}\left(C_{1}\right)$ in exactly one vertex, $a_{1}$ say, and such that $a_{0}$ is joined to all vertices in $K-a_{1}$. Thus $G$ contains a copy $H^{\prime}$ of $H$ which has exactly one vertex $x \in V_{G}\left(C_{1}\right)$ and whose other vertices lie in clusters belonging to $V\left(K-a_{1}\right) \cup\left\{a_{0}\right\}$. (Indeed, we can choose the vertices of $H^{\prime}$ lying in the same vertex class as $x$ in the cluster $a_{0}$ and the vertices lying in other vertex classes in the clusters belonging to $K-a_{1}$.) In fact, $G$ contains $|H|-1$ (say) disjoint such copies of $H$. Now suppose that $\left|V_{G}\left(C_{1}\right)\right| \equiv j \bmod |H|$. Then we take out $j$ disjoint such copies of $H$ in $G$ to achieve that $\left|V_{G}\left(C_{1}\right)\right|$ is divisible by $|H|$, i.e. we delete the vertices in these copies from the clusters they belong to. Next we consider the graphs $F_{1}:=F-V\left(C_{1}\right)$ and $R_{1}:=R-V_{R}\left(C_{1}\right)$ instead of $F$ and $R$. Claim 1 and (7) together imply that $\delta\left(R_{1}\right) \geq\left(1-\frac{1}{\ell-1+\xi}+\frac{\gamma}{4}\right)\left|R_{1}\right|$. Now suppose that $|\mathcal{C}| \geq 3$. Then similarly as in the proof of Claim 2 one can find a component $C_{2} \in \mathcal{C} \backslash\left\{C_{1}\right\}$, a copy $K^{\prime}$ of $K_{\ell}$ in $R_{1}$ and a vertex $a_{0}^{\prime} \in V\left(R_{1}\right) \backslash\left(V\left(K^{\prime}\right) \cup V_{R}\left(C_{2}\right)\right)$ such that $K^{\prime}$ meets $V_{R}\left(C_{2}\right)$ in exactly one vertex, $a_{2}$ say, and such that $a_{0}^{\prime}$ is joined to $K-a_{2}$. As before, we take out at most $|H|-1$ copies of $H$ in $G$ to achieve that $\left|V_{G}\left(C_{2}\right)\right|$ is divisible by $|H|$. As $|G|$ was divisible by $|H|$, we can continue in this fashion to achieve that $\left|V_{G}(C)\right|$ is divisible by $|H|$ for all components $C \in \mathcal{C}$. In this process, we have to take out at most $(|\mathcal{C}|-1)(|H|-1)$ copies of $H$ in $G$. Now we consider each component $C \in \mathcal{C}$ separately. By proceeding as in the connected case for each $C$ and taking out at most $(|C|-1)(|H|-1)$ further copies of $H$ in $G$ in each case, we can make $\left|V_{G}(B)\right|$ divisible by $|H|$ for each $B \in \mathcal{B}^{*}$. Hence, in total, we have taken out at most $(|\mathcal{C}|-1)(|H|-1)+\left(\left|\mathcal{B}^{*}\right|-|\mathcal{C}|\right)(|H|-1) \leq\left|\mathcal{B}^{*}\right||H|$ copies of $H$ in $G$.

5.5. Applying the Blow-up lemma. As described at the beginning of Section 5.4, we now consider each $B_{i}^{\prime} \subseteq R\left(i \leq k^{\prime}\right)$ where the vertices of $R$ are the modified clusters (i.e. they do not contain vertices lying in the copies of $H$ removed in Sections 5.3 and 5.4). For each $i \leq k^{\prime}$ let $G_{i}^{\prime}$ denote the subgraph of $G^{\prime}$ corresponding to $B_{i}^{\prime}$. Thus $G_{i}^{\prime}$ is the $\ell$-partite subgraph of $G^{\prime}$ whose $j$ th vertex class is the union of all the clusters lying in the $j$ th vertex class of $B_{i}^{\prime}(j=1, \ldots, \ell)$. In Section 5.4 we made $\left|G_{i}^{\prime}\right|=\left|V_{G}\left(B_{i}^{\prime}\right)\right|$ divisible by $|H|$ for each $i$. Moreover, in Section 5.3 we have removed at most $\beta L^{\prime}$ vertices from each cluster and in Section 5.4 we removed only a bounded number of further vertices. So altogether we removed at most $2 \beta L^{\prime}$ vertices from 
each cluster. Since $\beta \ll \lambda \ll 1-\xi$ and $\beta \ll \beta_{0}(H)$ by (5) and (6), we may apply Lemma 6 to conclude that the complete $\ell$-partite graph whose vertex classes are the same as the vertex classes of $G_{i}^{\prime}$ has a perfect $H$-packing. (Recall it was for this purpose that we chose the smallest vertex class of $B^{\prime}$ to be $\xi(1+\lambda)$ times as large as the others in the definition of $B^{\prime}$-and not just $\xi$ times as large.)

Moreover, each of the bipartite subgraphs of $G_{i}^{\prime}$ corresponding to an edge of $B_{i}^{\prime}$ is still $(6 \varepsilon, d / 6)$-superregular. Indeed, since all the copies of $H$ chosen in Section 5.3 avoid the red vertices of $G$, the removal of the vertices in all these copies results in bipartite subgraphs of $G_{i}^{\prime}$ which are still $(5 \varepsilon, d / 5)$-superregular. (This was already observed at the end of Section 5.2.) In Section 5.4 we only removed a bounded number of further vertices in each cluster. Thus after Section 5.4 the bipartite subgraphs of $G_{i}^{\prime}$ are still $(6 \varepsilon, d / 6)$-superregular. Hence, for each $i=1, \ldots, k^{\prime}$, we may apply the Blow-up lemma to find a perfect $H$-packing in $G_{i}^{\prime}$. Together with all the copies of $H$ chosen so far, all these $H$-packings form a perfect $H$-packing in $G$, as desired.

5.6. Algorithmic aspects of the proof. In this section, we point out those parts of the proof where the translation into an algorithm is nontrivial. Alon et al. [1] described an algorithm which finds the partition guaranteed by the Regularity lemma in time $O\left(n^{2.376}\right)$. An algorithmic version of the Blow-up lemma was found by Komlós, Sárközy and Szemerédi [16]. The algorithm runs in time $O\left(n^{3.376}\right)$. Note that the application of Theorem 3 to the reduced graph takes just constant time, as the order of the reduced graph is bounded. The partition of the vertex sets referred to in the paragraph after (8) can be found in time $O\left(n^{3}\right)$. This follows e.g. from $[2$, Ch. 15, Thm. 1.2] and its proof by considering the adjacency matrix of $G$. Also, each of the $O(n)$ copies of $H$ which we remove in Section 5.3 to incorporate the exceptional vertices can be found in time $O\left(n^{2}\right)$ using the 'greedy' argument described in [7, 18].

\section{REFERENCES}

[1] N. Alon, R.A. Duke, H. Lefmann, V. Rödl and R. Yuster, The algorithmic aspects of the Regularity lemma, J. Algorithms 16 (1994), 80-109.

[2] N. Alon and J. Spencer, The Probabilistic Method (2nd edition), Wiley-Interscience 2000.

[3] N. Alon and R. Yuster, H-factors in dense graphs, J. Combin. Theory B 66 (1996), 269-282.

[4] B. Bollobás, Modern Graph Theory, Graduate Texts in Mathematics 184, Springer-Verlag 1998.

[5] B.S. Baker, Approximation algorithms for NP-complete problems on planar graphs, Proc. 24th Ann. IEEE Symp. on Foundations of Computer Science (1983), 265-273.

[6] F. Berman, D. Johnson, T. Leighton, P.W. Shor and L. Snyder, Generalized planar matching, J. Algorithms 11 (1990), 153-184.

[7] R. Diestel, Graph Theory, Graduate Texts in Mathematics 173, Springer-Verlag 1997.

[8] P. Hell and D.G. Kirkpatrick, Scheduling, matching and colouring, Colloquia Math. Soc. Bolyai 25 (1978), $273-279$.

[9] P. Hell and D.G. Kirkpatrick, On the complexity of general graph factor problems, SIAM J. Computing 12 (1983), 601-609.

[10] C. Hurkens and A. Schrijver, On the size of systems of sets every $t$ of which have an SDR, with an application to the worst-case ratio of heuristics for packing problems, SIAM J. Disc. Math. 2 (1989), 68-72.

[11] V. Kann, Maximum bounded H-matching is MAX SNP-complete, Information Processing Letters 49 (1994), 309-318.

[12] K. Kawarabayashi, $K_{4}^{-}$-factors in a graph, J. Graph Theory 39 (2002), 111-128.

[13] J. Komlós, The Blow-up lemma, Comb. Probab. Comput. 8 (1999), 161-176.

[14] J. Komlós, Tiling Turán theorems, Combinatorica 20 (2000), 203-218.

[15] J. Komlós, G. N. Sárközy and E. Szemerédi, Blow-up lemma, Combinatorica 17 (1997), 109-123.

[16] J. Komlós, G. N. Sárközy and E. Szemerédi, An algorithmic version of the blow-up lemma, Random Struct. Alg. 12 (1998), 297-312.

[17] J. Komlós, G. N. Sárközy and E. Szemerédi, Proof of the Alon-Yuster conjecture, Discrete Math. 235 (2001), 255-269. 
[18] J. Komlós and M. Simonovits, Szemerédi's Regularity Lemma and its applications in graph theory, Bolyai Society Mathematical Studies 2, Combinatorics, Paul Erdös is Eighty (Vol. 2) (D. Miklós, V. T. Sós and T. Szőnyi eds.), Budapest (1996), 295-352.

[19] D. Kühn and D. Osthus, Perfect $K_{\ell}^{-}$packings in graphs, submitted.

[20] D. Kühn and D. Osthus, Critical chromatic number and perfect packings in graphs, in preparation.

[21] D. Kühn, D. Osthus and A. Taraz, Large planar subgraphs in dense graphs, J. Combin. Theory B, to appear.

[22] A. Shokoufandeh and Y. Zhao, Proof of a conjecture of Komlós, Random Struct. Alg. 23 (2003) 180-205.

Daniela Kühn \& Deryk Osthus

School of Mathematics

Birmingham University

Edgbaston

Birmingham B15 2TT

UK

E-mail addresses: $\{$ kuehn, osthus\}@maths.bham.ac.uk 